\title{
7-mm-long dental implants: retrospective clinical outcomes in medically compromised patients
}

\author{
Truc Thi Hoang Nguyen*, Mi Young Eo*, Yun Ju Cho, Hoon Myoung, Soung Min Kim \\ Department of Oral and Maxillofacial Surgery, Dental Research Institute, School of Dentistry, Seoul National University, Seoul, Korea
}

Abstract (J Korean Assoc Oral Maxillofac Surg 2019;45:260-266)

Objectives: Dental implants shorter than $8 \mathrm{~mm}$, called short dental implants (SDIs), have been considered to have a lower success rate than standard length implants. But recent studies have shown that SDIs have a comparable success rate, and implant diameter was more important for implant survival than implant length. Also, SDIs have many advantages, such as no need for sinus lifting or vertical bone grafting, which may limit use in medically compromised patients.

Materials and Methods: In this study, 33 patients with 47 implants 7-mm long were examined over the last four years. All patients had special medical history and were categorized into 3 groups: systemic disorders, such as diabetes mellitus (controlled or uncontrolled), mental disability, and uncontrolled hypertension; oral cancer ablation with reconstruction, with or without radiotherapy; diverse osteomyelitis, such as osteoradionecrosis and bisphosphonate-related osteonecrosis of the jaw. Most of these patients have insufficient residual bone quality due to mandible atrophy or sinus pneumatization.

Results: The implant diameters were $4.0(\mathrm{n}=38), 4.5(\mathrm{n}=8)$, and $5.0 \mathrm{~mm}(\mathrm{n}=1)$. Among the 47 implants placed, 2 implants failed before the last followup. The survival rate of 7-mm SDIs was $95.74 \%$ from stage I surgery to the last follow-up. Survival rates did not differ according to implant diameter. The mean marginal bone loss (MBL) at 3 months, 1 and 2 years was significantly higher than at implant installation, and the MBL at 1 year was also significantly higher than at 3 months. MBL at 1 and 2 years did not differ significantly.

Conclusion: Within the limitations of the present study, the results indicate that SDIs provide a reliable treatment, especially for medically compromised patients, to avoid sinus lifting or vertical bone grafting. Further, long-term follow-up is needed.

Key words: Dental implants, Survival rates, Alveolar bone loss

[paper submitted 2018. 6. 15 / revised 1st 2018. 8. 6, 2nd 2018. 8. 28 / accepted 2018. 8. 30]

\section{Introduction}

Current literature defines dental implants shorter than 8 $\mathrm{mm}$ as short dental implants (SDIs) ${ }^{1-4}$. SDIs were considered to have a lower success rate than standard length implants ${ }^{4,5}$. However, no distinct linear relationship between implant length and survival rate has been identified ${ }^{4,6}$, and recent studies have shown that SDIs have comparable success rates ${ }^{7-10}$.

\footnotetext{
Soung Min Kim

Department of Oral and Maxillofacial Surgery, School of Dentistry, Seoul National University, 101 Daehak-ro, Jongno-gu, Seoul 03080, Korea

TEL: +82-2-2072-0213 FAX: +82-2-766-4948

E-mail:smin5@snu.ac.kr

ORCID: https://orcid.org/0000-0002-6916-0489

*These authors contributed equally to this work as first authors.

(a) This is an open-access article distributed under the terms of the Creative Commons Attribution Non-Commercial License (http://creativecommons.org/ licenses/by-nc/4.0/), which permits unrestricted non-commercial use, distribution, and reproduction in any medium, provided the original work is properly cited.

Copyright (C) 2019 The Korean Association of Oral and Maxillofacial Surgeons. All rights reserved.
}

In some situations, the mechanical stress on a shorter implant might be lower than that on a longer implant ${ }^{11-13}$.

In some patients, pathologic conditions lead to insufficient residual bone quality. Planning implant therapy in these patients needs careful consideration to gain predictable results and avoid complications.(Fig. 1) In patients with insufficient bone volume, several procedures can be used such as maxillary sinus elevation, guided bone regeneration or edentulous ridge expansion; but they all involve prolonged healing time, higher morbidity, and high cost ${ }^{5}$. Recently, SDIs have been considered to be an alternative resolution to those conditions.

While the success and survival of SDIs has been widely investigated, studies on the survival rate of SDIs in medically compromised patients are limited. The purpose of this study was to determine the survival rate of SDIs in medically compromised patients. There are several systemic disorders that were approved to have the significance influence on dental implant treatment success. Although some authors did not 

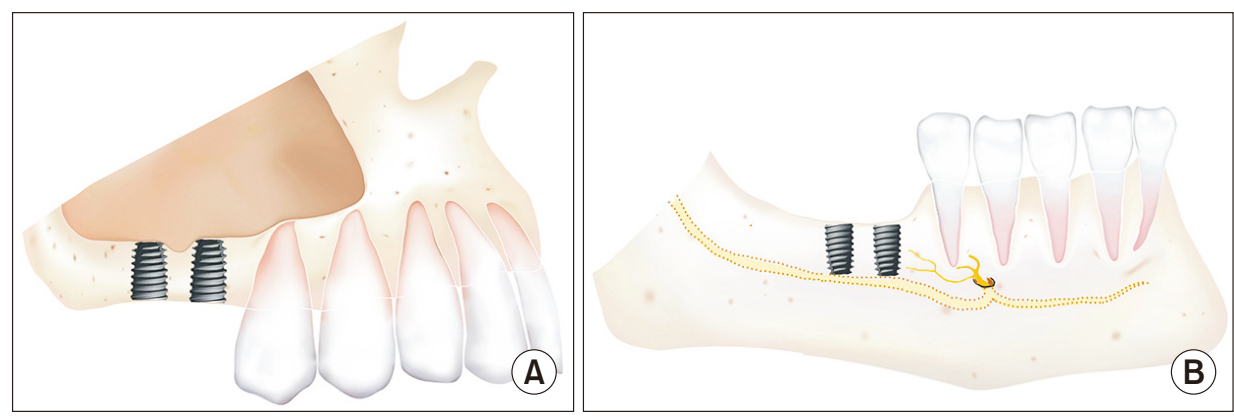

Fig. 1. Schematic drawing of short dental implant use in patients with insufficient residual bone volume due to sinus pneumatization (A) or mandible atrophy (B).

Truc Thi Hoang Nguyen et al: 7-mm-long dental implants: retrospective clinical outcomes in medically compromised patients. J Korean Assoc Oral Maxillofac Surg 2019 find the negative effect of diabetes mellitus to implantation outcomes $^{14}$, other studies found statistically significant relationship of implant failure and diabetes mellitus (controlled and uncontrolled) $)^{15}$. The compromised condition of gingival microvascular in diabetes patients may affect wound healing and increasing the risk of infection ${ }^{15}$.

The effect of uncontrolled hypertension condition on survival rate of dental implant is under controversy ${ }^{16}$. The risk of cardiovascular complications and renal failure in uncontrolled hypertension patients are well established ${ }^{17}$. These complications may affect the ossteointegration and change of alveolar bone level. Result of some studies show that patients with cardiovascular disease had increased peri-implant bone loss and peri-implantitis ${ }^{18}$.

In this retrospective study, patients who had special medical histories were categorized into 3 groups: systemic disorders, such as uncontrolled or controlled diabetes mellitus, mental disability, and uncontrolled hypertension; oral cancer ablation with reconstruction, with or without radiotherapy; diverse osteomyelitis, such as osteoradionecrosis and bisphosphonate-related osteonecrosis of the jaw (BRONJ). Most of these patients have insufficient residual bone quality due to mandible atrophy or sinus pneumatization. Marginal bone loss (MBL) is a generally accepted parameter for evaluating bone response around a dental implant ${ }^{19}$. Therefore, we evaluated MBL of SDIs on panoramic radiographs taken at implant installation, 3 month, 1-year and 2-years follow-up visits.

Thirty-three patients with forty-seven implants that were 7-mm long were examined during the last 4 years. The implant diameters were $4.0(\mathrm{n}=38), 4.5(\mathrm{n}=8)$, and $5.0 \mathrm{~mm}(\mathrm{n}=1)$. We analyzed SDI survival rate in the 3 patient groups, evaluated MBL, and discussed clinical implications.
Table 1. Distribution of patients in medical history groups

\begin{tabular}{cc}
\hline Medical history groups & No. of patients \\
\hline Systemic disorder (hypertension, diabetes, mental & 9 \\
disability, heart disease, etc.) & \\
Oral cancer ablation with reconstruction (with or & 13 \\
$\begin{array}{l}\text { without radiotherapy) } \\
\text { Osteomyelitis }\end{array}$ & 16 \\
\hline
\end{tabular}

${ }^{1}$ There are five patients had more than one special medical history including: osteomyelitis condition on a medical history of systemic disorder or previously oral cancer treatment.

Truc Thi Hoang Nguyen et al: 7-mm-long dental implants: retrospective clinical outcomes in medically compromised patients. J Korean Assoc Oral Maxillofac Surg 2019

\section{Materials and Methods}

\section{Patients data}

Thirty-three patients with SDIs placed from January 2015 to March 2018 at the Department of Oral and Maxillofacial Surgery at Seoul National University Dental Hospital (Seoul, Korea) were evaluated in this study.

Sample was chosen according to the following inclusion criterions: (1) medically compromised patients that belong to at least one of 3 groups: systemic disorders, such as diabetes mellitus (controlled or uncontrolled), mental disability, and uncontrolled hypertension; oral cancer ablation with reconstruction that associated to implantation sites, with or without radiotherapy; and diverse osteomyelitis such as osteoradionecrosis or BRONJ (Table 1); (2) patients were treated with the installation of internal submerged tapered Luna (Shinhung, Seoul, Korea) and internal non-submerged Stella (Shinhung) sand blasted and acid etched (S\&E) SDIs; and (3) patients didn't receive any bone augmentation at the implantation site. All the selected patients have insufficient residual bone quality due to mandible atrophy or sinus pneumatization.

All implants were placed through 1- or 2-stage procedures with a 3- to 6-month interval. Under local anesthesia, implants were installed according to the Luna and Stella implant surgical protocol by a single maxillofacial implant surgeon. 

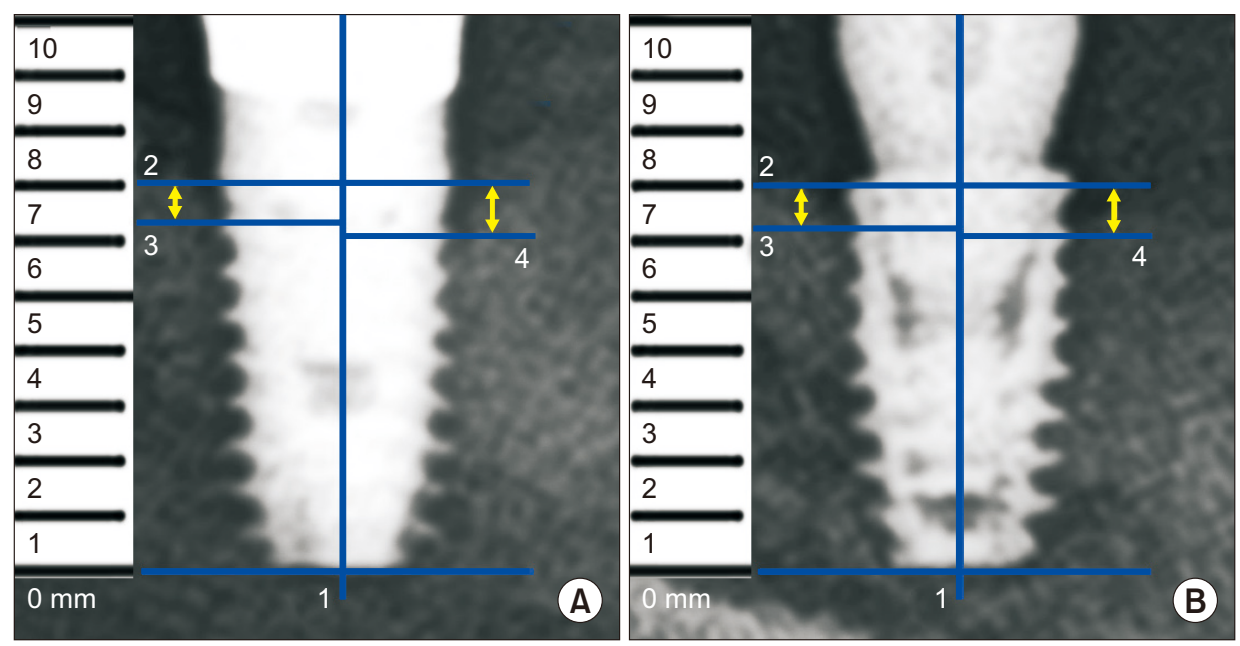

Fig. 2. Marginal bone loss (MBL) evaluation used in this study, reference lines were drawn to calculate bone loss on the mesial and distal sides of implant: longitudinal implant axis ("1"), horizontal line at the most coronal level of the implant collar ("2"), horizontal lines at the most coronal level of bone-to-implant contact at the mesial and distal sites (" 3 " and "4"). MBL measurement in a Stella implant (Shinhung; A) and Luna implant (Shinhung; B).

Truc Thi Hoang Nguyen et al: 7-mm-long dental implants: retrospective clinical outcomes in medically compromised patients. J Korean Assoc Oral Maxillofac Surg 2019

Table 2. Short dental implant installation locations

\begin{tabular}{lccc}
\hline & Maxilla & Mandible & Total \\
\hline Anterior & 2 & 6 & 8 \\
Posterior & 17 & 22 & 39 \\
Total & 19 & 28 & 47 \\
\hline
\end{tabular}

Truc Thi Hoang Nguyen et al: 7-mm-long dental implants: retrospective clinical outcomes in medically compromised patients. J Korean Assoc Oral Maxillofac Surg 2019

All implants initially achieved good primary stability. A panoramic radiograph was taken of all cases after implant surgery. This retrospective data analysis was approved by the Institutional Review Board of Seoul National University (SD20180022).

\section{Marginal bone loss evaluation}

MBL was determined from panoramic radiographs and expressed as the distance from the implant shoulder to the most coronal bone-to-implant contact on the mesial and distal sides of the implant. The relationship between the implant shoulder and marginal bone was measured mesially and distally by using reference lines including a line along the longitudinal implant axis, a horizontal line at the most coronal level of the implant shoulder, and two horizontal lines at the most coronal level of bone-to-implant contact mesially and distally ${ }^{20}$. MBL was evaluated on panoramic radiographs taken at implant placement, and at 3 months, 1 year, and 2 years follow-up visit. The MBL was measured at the same magnification on all installation and follow-up radiographs. Each aspect was measured 3 times, and the average was recorded.(Fig. 2) The change in MBL from installation at follow-up visits and changes between consecutive visits were calculated. A failed implant was considered as a lost or mobile implant or severe
Table 3. Prosthesis data of 45 success implants

\begin{tabular}{lc}
\hline \multicolumn{1}{c}{ Types of prosthesis } & No. of implants \\
\hline Single crown & 19 \\
Multiple fixed prosthesis & 22 \\
Removable overdenture & 4 \\
\hline
\end{tabular}

Truc Thi Hoang Nouyen et al: 7-mm-long dental implants: retrospective clinical outcomes in medically compromised patients. J Korean Assoc Oral Maxillofac Surg 2019

peri-implantitis that required prompt removal.

\section{Statistical analysis}

The collected data included descriptive and quantitative data. IBM SPSS Statistics software (ver. 25.0; IBM Corp., Armonk, NY, USA) was used for statistical analyses. Descriptive statistics were used to analyze and calculate the distributions of qualitative variables. For analyzing quantitative variables to assess $\mathrm{MBL}$, mean and standard deviation were calculated. We evaluated MBL data using the Shapiro-Wilk normality test. Data review and statistical analysis were performed by a single researcher (T.T.H.N.).

\section{Results}

Among the 33 patients, 11 were male, and 22 were female. Patient ages at installation ranged from 30 to 82 years and averaged 62 years. In total 47 implants were installed with diameters of $4.0(\mathrm{n}=38), 4.5(\mathrm{n}=8)$, and $5.0 \mathrm{~mm}(\mathrm{n}=1)$. Of the 47 implants, 6 were Stella implants, and 41 were Luna implants. Nineteen implants were installed in the maxilla and twenty-eight in the mandible.(Table 2) The follow-up periods ranged from 7 to 36 months with an average of 15 months.

In total 45 success implants, there were 19 implants sup- 
porting single crown restorations, 22 implants supporting multiple fixed prostheses, 4 implants supporting removable overdentures.(Table 3)

\section{Survival rate}

Among the 47 implants placed, 2 implants failed before the last follow-up. The survival rate of 7-mm SDIs was $95.74 \%$ from stage I surgery to the last follow-up. The survival rates of 4.0-mm-diameter implants was 94.74\%, 4.5-mm-diameter implants was $100 \%$, and 5.0-mm-diameter implants was $100 \%$.(Table 4) Both failed implants were $4.0 \mathrm{~mm}$ in diameter in a patient who had oral cancer and underwent reconstruction. Survival rates for the three diameters did not differ significantly $(P=0.069 ; P>0.05)$.

\section{Marginal bone loss}

The mean MBL between implant installation and 1 month on the mesial and distal aspects was $0.34 \pm 0.47 \mathrm{~mm}$ and $0.53 \pm 0.57 \mathrm{~mm}$, between installation and 1 year on the mesial and distal aspects was $0.53 \pm 0.58 \mathrm{~mm}$ and $0.67 \pm 0.56 \mathrm{~mm}$, respectively. It was $0.58 \pm 0.60 \mathrm{~mm}$ and $0.71 \pm 0.60 \mathrm{~mm}$, respectively, between implant installation and 2 years. Between 3 month and 1 year, the mean MBL on mesial and distal aspects increased by $0.19 \pm 0.24$ and $0.14 \pm 0.02$; between 1 and 2 years, it was $0.05 \pm 0.12$ and $0.04 \pm 0.05 \mathrm{~mm}$, respectively. The mean MBL at 3 months and 1 and 2 years was significantly higher than at implant installation. The mean MBL at 1 year

Table 4. Survival rate of the 47 short dental implants

\begin{tabular}{ccc}
\hline Diameter $(\mathrm{mm})$ & Success/fail (total) & Survival rate $(\%)$ \\
\hline 4.0 & $36 / 2(38)$ & 94.74 \\
4.5 & $8 / 0(8)$ & 100 \\
5.0 & $1 / 0(1)$ & 100 \\
Total & $45 / 2(47)$ & 95.74
\end{tabular}

Survival rates did not differ significantly among the three diameter groups $(P=0.069)$.

Truc Thi Hoang Nguyen et al: 7-mm-long dental implants: retrospective clinical outcomes in medically compromised patients. J Korean Assoc Oral Maxillofac Surg 2019 was also significantly higher than at 3 months. The MBL at 1 and 2 years did not differ significantly $(P<0.05)$. (Table 5)

\section{Discussion}

Using the longest possible implants was considered conventional therapy based on the principle that longer implants would have higher survival rates and a more favorable prognosis $^{21}$. However, in many clinical conditions, long implants were limited or unfavorable due to insufficient bone volume, maxillary sinus pneumatization, and inferior alveolar nerve canal position.

The indication for SDIs remains controversial because of challenges such as less bone-to-implant contact due to reduced implant surface, more crestal bone resorption due to a smaller surface over which to distribute forces, and increased crown-to-implant (C/I) ratio ${ }^{11,19}$. The technique for installing SDIs involves some considerations. First, the direction is easily distorted when drilling because the hole made for SDIs is shallower than for longer implants. Therefore, placing an SDI requires more skill. Second, the hole made by the counterbore should not be too deep because of the short fixture. Third, the implant-supported restoration should not be too large.

Despite these considerations, SDI has many advantages to both the patient and surgeon. Using SDI avoids bone grafting and nerve transposition, reduces donor site morbidity for autogenous bone grafting, reduces nerve damage for nerve transposition, and, therefore, reduces treatment time and cost and patient discomfort ${ }^{22}$. SDI can help decrease the possibility of contact with adjacent tooth roots, lower the risk of surgical paresthesia, reduce bone overheating, and lower the risk of bone graft exposure, which brings significant advantages to implant therapy for medical compromised patients.

We present 3 cases of SDI placement, one from each medical condition group. Case 1 was a 76-year-old female who had squamous cell cancer and was treated with maxillary mass resection and radiotherapy on the left, and then underwent radical neck dissection and radiotherapy due to

Table 5. Marginal bone loss (MBL) evaluation on the mesial and distal aspects of short dental implants at 3 months, 1 year, and 2 years

\begin{tabular}{|c|c|c|c|c|}
\hline & \multicolumn{2}{|c|}{ MBL from installation $(\mathrm{mm})^{1}$} & \multicolumn{2}{|c|}{ MBL from previous visit $(\mathrm{mm})^{2}$} \\
\hline & Mesial & Distal & Mesial & Distal \\
\hline 3 months & $0.34 \pm 0.47$ & $0.53 \pm 0.57$ & $0.34 \pm 0.47$ & $0.53 \pm 0.57$ \\
\hline 1 year & $0.53 \pm 0.58$ & $0.67 \pm 0.56$ & $0.19 \pm 0.24$ & $0.14 \pm 0.02$ \\
\hline 2 years & $0.58 \pm 0.60$ & $0.71 \pm 0.60$ & $0.05 \pm 0.12$ & $0.04 \pm 0.05$ \\
\hline
\end{tabular}

${ }^{1}$ Mean MBL at 3 months, 1 year, and 2 years was significantly higher than at implant installation $(P<0.05)$.

${ }^{2}$ Mean MBL at 1 year was significantly higher than at 3 months. MBL at 1 and 2 years did not differ significantly $(P<0.05)$.

Truc Thi Hoang Nguyen et al: 7-mm-long dental implants: retrospective clinical outcomes in medically compromised patients. J Korean Assoc Oral Maxillofac Surg 2019 
right neck metastasis. An implant was planned for the edentulous region of the right posterior mandible. After considering mandible atrophy and proximity to the inferior alveolar nerve, a $4.5 \mathrm{~mm} \times 7 \mathrm{~mm}$ Stella implant was placed. The implant achieved good stability and bone integration after loading and showed acceptable MBL at 3 years.(Fig. 3) Case 2 is a 72-year-old male with osteomyelitis and a history of hypertension and diabetes. A $4 \mathrm{~mm} \times 7 \mathrm{~mm}$ Stella implant was installed in the 37 tooth site. The implant showed good stability and low MBL after loading and at 1 year.(Fig. 4) Case 3 is a 67-year-old female who had hypertension and osteomyelitis in the posterior right mandible. A previous implant installed in the 37 tooth position failed due to bone resorption. In addition, the edentulous posterior of the right maxilla also had insufficient bone and sinus pneumatization. Therefore, in planning the implant therapy, a $4 \mathrm{~mm} \times 7 \mathrm{~mm}$ Luna implant was chosen for the 16 position, and a $4.5 \mathrm{~mm} \times 7 \mathrm{~mm}$ Stella implant was chosen for the 47 tooth position after removal of the failed implant. The two implants showed good stability and acceptable MBL on follow-up examination.(Fig. 5)

Of the 47 implants, two failed, and the survival rate was $95.74 \%$. The two failed implants belonged to a patient with oral cancer who was treated with mandibular resection and reconstruction. The postoperative bone had insufficient volume and unfavorable quality. Dental implant treatment has few absolute contraindications, and the impact of health risks on implant outcome remains unclear due to the scarcity of prospective studies ${ }^{19}$. However, studies have shown a negative impact of bisphosphonates on implant success ${ }^{19}$. In oral cancer patients, a lack of residual bone following resection makes placing implants in an ideal position difficult ${ }^{23,24}$. Considering that all patients were medically compromised,
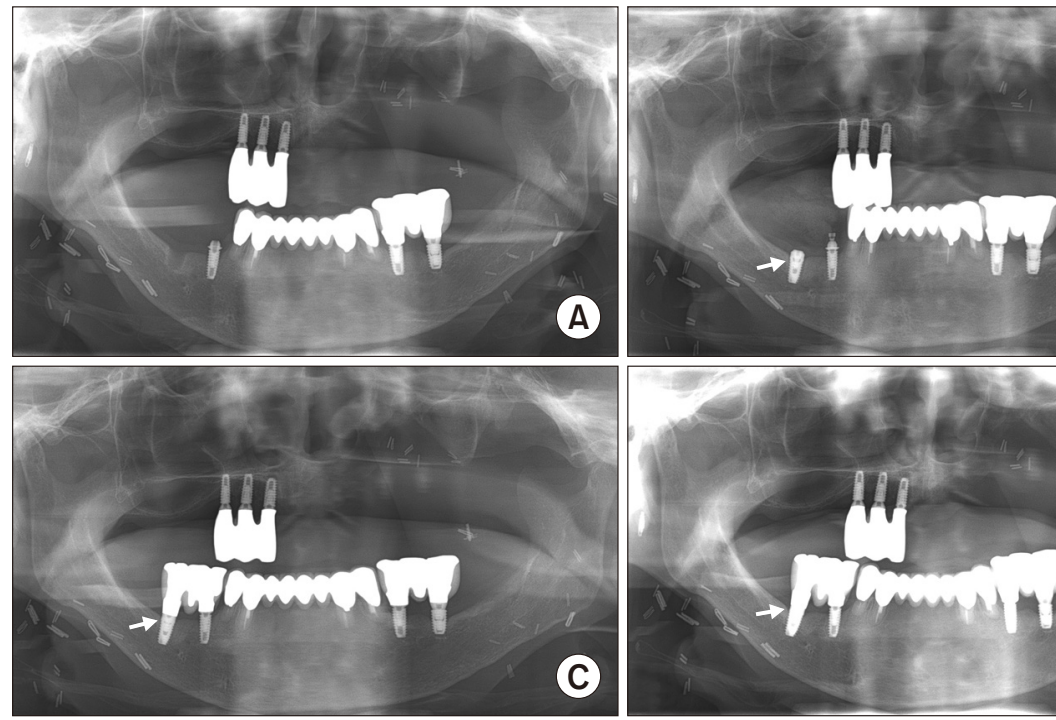

c
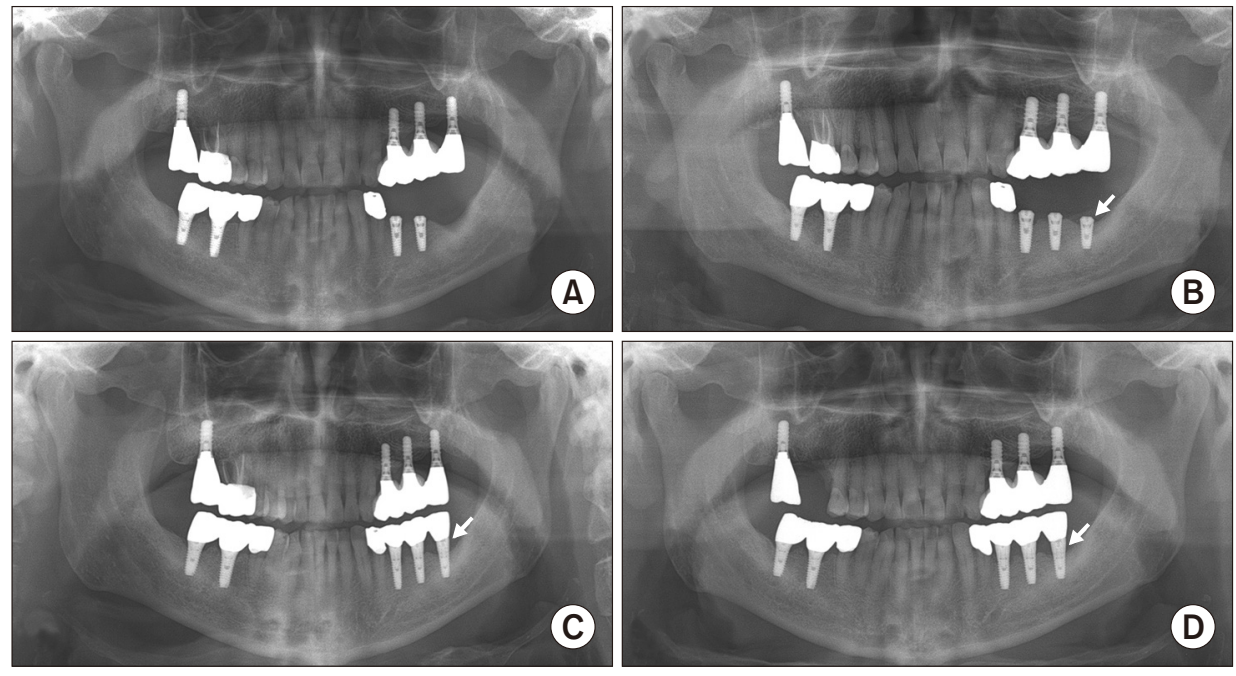

Fig. 3. Panoramic radiograms of a 76-year-old female who had squamous cell cancer, treated with maxillary mass resection and radiotherapy on the left, radical neck dissection and radiotherapy due to neck metastasis on the right (A). A $4.5 \mathrm{~mm} \times 7 \mathrm{~mm}$ Stella (Shinhung) was installed in the 47 tooth position (arrow; B). The implant achieved good stability and bone integration, and showed acceptable bone loss at 3 years (arrows; C, D).

Truc Thi Hoang Nguyen et al: 7-mm-long dental implants: retrospective clinical outcomes in medically compromised patients. J Korean Assoc Oral Maxillofac Surg 2019

Fig. 4. Panoramic radiogram of a 72-year-old male with osteomyelitis and a history of hypertension and diabetes. A. Preoperation radiogram. B. A $4 \mathrm{~mm} \times 7 \mathrm{~mm}$ Stella (Shinhung) implant was installed in the 37 tooth position (arrow). C, D. The implant achieved good stability and bone integration, and showed acceptable bone loss at 1 year (arrows).

Truc Thi Hoang Nguven et al: 7-mm-long dental implants: retrospective clinical outcomes in medically compromised patients. J Korean Assoc Oral Maxillofac Surg 2019 

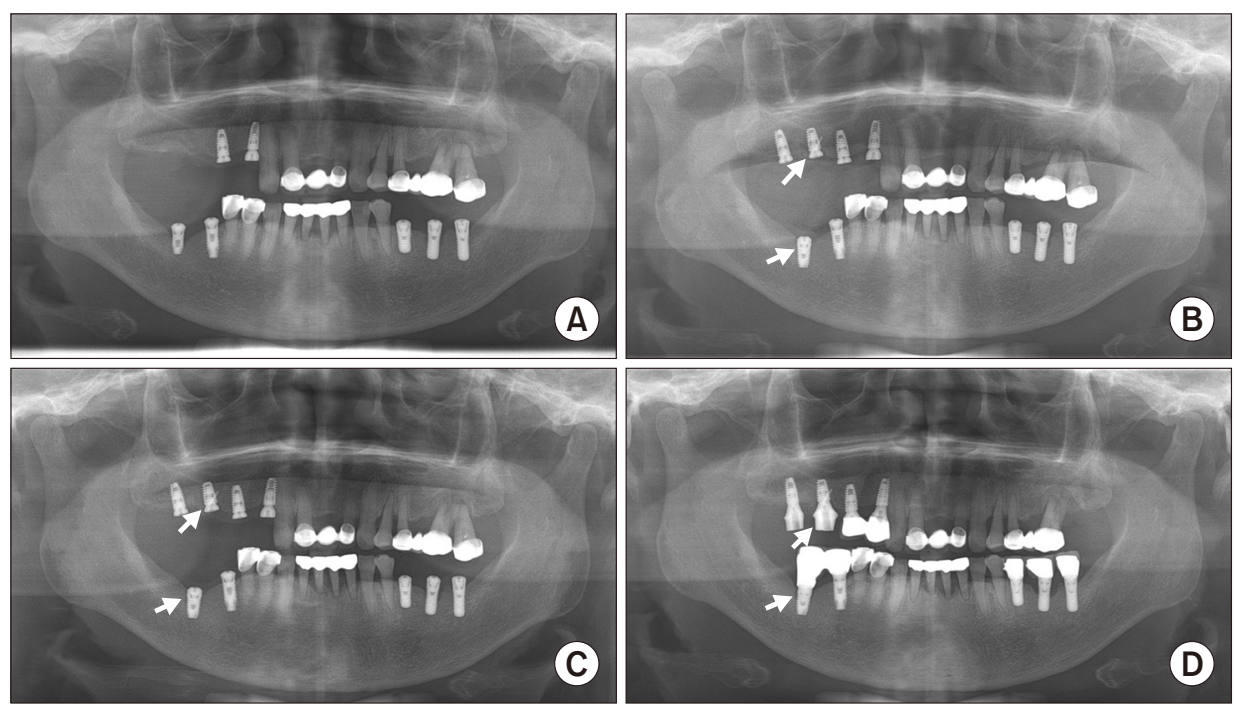

Fig. 5. Panoramic radiogram of a 67-year-old female who had hypertension and osteomyelitis in the posterior right mandible. A. A previous implant installed in the 37 tooth position failed due to bone resorption. B. A $4 \mathrm{~mm} \times 7$ $\mathrm{mm}$ Luna (Shinhung) implant was placed at the 16 tooth position (arrow), and a $4.5 \mathrm{~mm} \times 7 \mathrm{~mm}$ Stella (Shinhung) implant was placed at the 47 tooth position (arrow) after removal of the failed implant. C, D. The two implants showed good stability and acceptable bone loss on follow-up examination (arrows).

Truc Thi Hoang Nguyen et al: 7-mm-long dental implants: retrospective clinical outcomes in medically compromised patients. J Korean Assoc Oral Maxillofac Surg 2019 including cancer and BRONJ, the survival rate of 7-mmlong implants in the general population would be higher than in this study. The failed implants in this study were placed adventurously in alveolar bone, which had insufficient height and had been involved in cancer treatment and reconstruction surgery. Most implant failures were reported early, during the healing phase at abutment connection ${ }^{25-29}$.

To guarantee long-term clinical results, maintaining stable marginal bone is more critical with $\mathrm{SDIs}^{18}$. MBL is a generally accepted parameter to evaluate the bone response around a dental implant. Originally, a mean $\mathrm{MBL}$ of $\geq 1.5 \mathrm{~mm}$ in the first year and an MBL of $\geq 0.2 \mathrm{~mm}$ per year afterward was considered a threshold for implant success ${ }^{20,30}$. Randomized, controlled studies $^{31}$ on SDIs in the posterior maxilla had an MBL from 1.02 to $0.1 \mathrm{~mm}$. In this study, the MBL results on the mesial and distal aspects after 1 year were $0.53 \pm 0.58$ $\mathrm{mm}$ and $0.67 \pm 0.56 \mathrm{~mm}$, respectively, and $0.58 \pm 0.60 \mathrm{~mm}$ and $0.71 \pm 0.60 \mathrm{~mm}$, respectively, after 2 years. These MBL results are within the success threshold ${ }^{20}$; however, long-term follow-up is needed.

\section{Conclusion}

The present study showed comparable survival rates of SDIs in medically compromised patients to the conventional implants in a healthy population. In addition, the stability of marginal bone around an SDI in these patients was acceptable in comparison with MBL in healthy patients. The results suggest that placing an SDI is a reliable treatment option, especially for medically compromised patients, and can be an alternative when sinus lifting or vertical bone grafting should be avoided. Further, long-term follow-up and evaluation of SDIs in these patients is needed.

\section{ORCID}

Truc Thi Hoang Nguyen, https://orcid.org/0000-0002-8667-6698

Mi Young Eo, https://orcid.org/0000-0001-7055-9924

Yun Ju Cho, https://orcid.org/0000-0002-1818-5280

Hoon Myoung, https://orcid.org/0000-0002-9984-8479

Soung Min Kim, https://orcid.org/0000-0002-6916-0489

\section{Authors' Contributions}

All authors read and approved the final manuscript. T.T.H.N. read and wrote the manuscript, M.Y.E. prepared retrospective data and wrote the manuscript, Y.J.C. prepared all figures and references, H.M. revised and corrected the manuscript, and S.M.K. designed and wrote the entire article.

\section{Acknowledgements}

This study was supported by Basic Science Research Program through the National Research Foundation of Korea funded by the Ministry of Education (2017R1D1A1B03036054).

\section{Ethics Approval and Consent to Participate}

This retrospective data analysis was approved by the Institutional Review Board of Seoul National University (SD20180022). 


\section{Conflict of Interest}

No potential conflict of interest relevant to this article was reported.

\section{References}

1. Renouard F, Nisand D. Impact of implant length and diameter on survival rates. Clin Oral Implants Res 2006;17 Suppl 2:35-51.

2. Jain N, Gulati M, Garg M, Pathak C. Short implants: new horizon in implant dentistry. J Clin Diagn Res 2016;10:ZE14-7.

3. Al-Hashedi AA, Taiyeb Ali TB, Yunus N. Short dental implants: an emerging concept in implant treatment. Quintessence Int 2014:45:499-514.

4. Nisand D, Renouard F. Short implant in limited bone volume. Periodontol 2000 2014;66:72-96.

5. Sennerby L, Roos J. Surgical determinants of clinical success of osseointegrated oral implants: a review of the literature. Int J Prosthodont 1998;11:408-20.

6. Wyatt CC, Zarb GA. Treatment outcomes of patients with implantsupported fixed partial prostheses. Int J Oral Maxillofac Implants 1998;13:204-11.

7. Srinivasan M, Vazquez L, Rieder P, Moraguez O, Bernard JP, Belser UC. Efficacy and predictability of short dental implants $(<8$ $\mathrm{mm})$ : a critical appraisal of the recent literature. Int J Oral Maxillofac Implants 2012;27:1429-37.

8. Karthikeyan I, Desai SR, Singh R. Short implants: a systematic review. J Indian Soc Periodontol 2012;16:302-12.

9. Lemos CA, Ferro-Alves ML, Okamoto R, Mendonça MR, Pellizzer EP. Short dental implants versus standard dental implants placed in the posterior jaws: a systematic review and meta-analysis. J Dent 2016;47:8-17.

10. Benlidayi ME, Ucar Y, Tatli U, Ekren O, Evlice B, Kisa HI, et al. Short implants versus standard implants: midterm outcomes of a clinical study. Implant Dent 2018;27:95-100.

11. Esfahrood ZR, Ahmadi L, Karami E, Asghari S. Short dental implants in the posterior maxilla: a review of the literature. J Korean Assoc Oral Maxillofac Surg 2017;43:70-6.

12. Pierrisnard L, Renouard F, Renault P, Barquins M. Influence of implant length and bicortical anchorage on implant stress distribution. Clin Implant Dent Relat Res 2003;5:254-62.

13. Anitua E, Tapia R, Luzuriaga F, Orive G. Influence of implant length, diameter, and geometry on stress distribution: a finite element analysis. Int J Periodontics Restorative Dent 2010;30:89-95.

14. Chen H, Liu N, Xu X, Qu X, Lu E. Smoking, radiotherapy, diabetes and osteoporosis as risk factors for dental implant failure: a meta-analysis. PLoS One 2013;8:e71955.

15. Moy PK, Medina D, Shetty V, Aghaloo TL. Dental implant failure rates and associated risk factors. Int J Oral Maxillofac Implants 2005;20:569-77.

16. Guobis Z, Pacauskiene I, Astramskaite I. General diseases influence on peri-implantitis development: a systematic review. J Oral
Maxillofac Res 2016;7:e5.

17. Liddelow G, Klineberg I. Patient-related risk factors for implant therapy. A critique of pertinent literature. Aust Dent J 2011;56:41726; quiz 441.

18. Renvert S, Aghazadeh A, Hallström H, Persson GR. Factors related to peri-implantitis - a retrospective study. Clin Oral Implants Res 2014;25:522-9.

19. Kasai T, Pogrel MA, Hossaini M. The prognosis for dental implants placed in patients taking oral bisphosphonates. J Calif Dent Assoc 2009;37:39-42.

20. Galindo-Moreno P, León-Cano A, Ortega-Oller I, Monje A, O Valle F, Catena A. Marginal bone loss as success criterion in implant dentistry: beyond $2 \mathrm{~mm}$. Clin Oral Implants Res 2015;26:e28-34.

21. Diz P, Scully C, Sanz M. Dental implants in the medically compromised patient. J Dent 2013;41:195-206.

22. Grant BT, Pancko FX, Kraut RA. Outcomes of placing short dental implants in the posterior mandible: a retrospective study of 124 cases. J Oral Maxillofac Surg 2009;67:713-7.

23. Barrowman RA, Wilson PR, Wiesenfeld D. Oral rehabilitation with dental implants after cancer treatment. Aust Dent J 2011;56:160-5.

24. Renouard F, Nisand D. Short implants in the severely resorbed maxilla: a 2-year retrospective clinical study. Clin Implant Dent Relat Res 2005; 7 Suppl 1:104-10.

25. Jemt T, Lindén B, Lekholm U. Failures and complications in 127 consecutively placed fixed partial prostheses supported by Brånemark implants: from prosthetic treatment to first annual checkup. Int J Oral Maxillofac Implants 1992;7:40-4

26. Becker W, Becker BE, Alsuwyed A, Al-Mubarak S. Long-term evaluation of 282 implants in maxillary and mandibular molar positions: a prospective study. J Periodontol 1999;70:896-901.

27. Lee JH, Frias V, Lee KW, Wright RF. Effect of implant size and shape on implant success rates: a literature review. J Prosthet Dent 2005;94:377-81.

28. Morand M, Irinakis T. The challenge of implant therapy in the posterior maxilla: providing a rationale for the use of short implants. $\mathrm{J}$ Oral Implantol 2007;33:257-66.

29. Mohajerani H, Roozbayani R, Taherian S, Tabrizi R. The risk factors in early failure of dental implants: a retrospective study. J Dent (Shiraz) 2017;18:298-303.

30. Bratu E, Chan HL, Mihali S, Karancsi O, Bratu DC, Fu JH, et al. Implant survival rate and marginal bone loss of 6-mm short implants: a 2-year clinical report. Int J Oral Maxillofac Implants 2014;29:1425-8

31. Monje A, Chan HL, Fu JH, Suarez F, Galindo-Moreno P, Wang HL. Are short dental implants $(<10 \mathrm{~mm})$ effective? A meta-analysis on prospective clinical trials. J Periodontol 2013;84:895-904.

How to cite this article: Nguyen TTH, Eo MY, Cho YJ, Myoung H, Kim SM. 7-mm-long dental implants: retrospective clinical outcomes in medically compromised patients. J Korean Assoc Oral Maxillofac Surg 2019;45:260-266. https://doi.org/10.5125/ jkaoms.2019.45.5.260 